\title{
THE MOMENT MAP FOR A MULTIPLICITY FREE ACTION
}

\author{
CHAL BENSON, JOE JENKINS, RONALD L. LIPSMAN, AND GAIL RATCLIFF
}

\begin{abstract}
Let $K$ be a compact connected Lie group acting unitarily on a finite-dimensional complex vector space $V$. One calls this a multiplicity-free action whenever the $K$-isotypic components of $\mathbb{C}[V]$ are $K$-irreducible. We have shown that this is the case if and only if the moment map $\tau: V \rightarrow k^{*}$ for the action is finite-to-one on $K$-orbits. This is equivalent to a result concerning Gelfand pairs associated with Heisenberg groups that is motivated by the Orbit Method. Further details of this work will be published elsewhere.
\end{abstract}

\section{INTRODUCTION}

1.1. Gelfand pairs. Suppose that $G$ is a Lie group and that $K$ is a compact subgroup of $G$. One says that $K \subset G$ is a Gelfand pair when the algebra $L^{1}(G / / K)$ of integrable $K$-bi-invariant functions on $G$ is commutative under convolution. This note concerns a special class of Gelfand pairs that are associated with Heisenberg groups. Let $V$ be a finite-dimensional complex vector space with Hermitian structure $\langle\cdot, \cdot\rangle:=(\cdot, \cdot)+i \omega(\cdot, \cdot)$, and form the associated Heisenberg group $H_{V}:=V \times \mathbb{R}$ with product $(z, t)\left(z^{\prime}, t^{\prime}\right):=$ $\left(z+z^{\prime}, t+t^{\prime}-\frac{1}{2} \omega\left(z, z^{\prime}\right)\right)$. Let $K$ be a compact connected Lie group acting on $V$ via some unitary representation $K \times V \rightarrow V,(k, z) \mapsto k z$. We obtain an action of $K$ on $H_{V}$ by automorphisms $k \cdot(z, t):=(k z, t)$ and form the semidirect product $G:=K \ltimes H_{V}$. In this setting, $L^{1}(G / / K)$ is naturally identified with the algebra $L_{K}^{1}\left(H_{V}\right)$ of integrable $K$-invariant functions on the Heisenberg group. We will say that $\left(K, H_{V}\right)$ is a Gelfand pair when this algebra is commutative.

A fundamental result due to I. M. Gelfand asserts that $K \subset G$ is a Gelfand pair if and only if each irreducible unitary representation $\pi$ of $G$ has at most a one-dimensional space of $K$-fixed vectors [5]. The $K$-spherical representations are those that have nonzero $K$-fixed vectors. By using the Mackey machine to describe the unitary dual of $K \ltimes H_{V}$, one obtains an interesting specialization of this criterion to pairs of the form $K \subset K \ltimes H_{V}$. Namely, $\left(K, H_{V}\right)$ is a Gelfand pair if and only if the action of $K$ on $V$ is multiplicity-free [2, 3]. The latter condition means that each irreducible representation of $K$ occurs at most once in the representation $k \cdot p(z):=p\left(k^{-1} z\right)$ of $K$ on $\mathbb{C}[V]$. Multiplicity-free actions are of considerable interest in their own right, but are usually studied in the setting of reductive complex algebraic groups. One can pass to this setting by complexifying $K$. The irreducible multiplicity-free actions of connected reductive complex algebraic groups were classified by V. Kac [10]. This yields a

Received by the editors January 13, 1994.

1991 Mathematics Subject Classification. Primary 22E30, 43A55.

Key words and phrases. Gelfand pairs, Heisenberg group, Orbit Method, moment map.

All authors were supported in part by the National Science Foundation. 
complete classification of the Gelfand pairs $\left(K, H_{V}\right)$ where $K$ acts irreducibly on $V[2]$.

The literature contains various results relating multiplicities of Lie group representations to geometric properties of coadjoint orbits. See for example $[4,6,7,8,13]$. These results motivate a conjecture that provides a geometric formulation of Gelfand's criterion for Gelfand pairs $\left(K, H_{V}\right)$. Let $\mathfrak{k}, \mathfrak{g}$ denote the Lie algebras of $K, G=K \ltimes H_{V}$, and let $\mathfrak{k}^{*}, \mathfrak{g}^{*}$ be their duals. The Orbit Method (also called "Geometric Quantization") yields one-to-one correspondences between the unitary duals $\widehat{K}, \widehat{G}$ of $K, G$ and the integral orbits in $\mathfrak{k}^{*}, \mathfrak{g}^{*}$ for the coadjoint actions of $K, G$. (See [8] and [12] respectively.) Let $\pi: \mathfrak{g}^{*} \rightarrow \mathfrak{k}^{*}$ be the restriction map and $\mathfrak{k}^{\perp}:=\pi^{-1}(\{0\})$. If $\xi \in \mathfrak{g}^{*}$ and $\mathscr{O}_{\xi}^{G}:=\operatorname{Ad}^{*}(G) \xi$ is an integral orbit corresponding to $\rho_{\xi} \in \widehat{G}$, then the multiplicity of the trivial representation of $K$ in $\left.\rho_{\xi}\right|_{K}$ (i.e. the dimension of the space of $K$-fixed vectors) should be related to the number of $K$-orbits in the intersection $\mathfrak{k}^{\perp} \cap \mathcal{O}_{\xi}^{G}$.

Orbit Conjecture. Let $K$ be a compact connected Lie group acting unitarily on $V$. Then $\left(K, H_{V}\right)$ is a Gelfand pair if and only if

$$
\text { for every } \xi \in \mathfrak{k}^{\perp}, \mathscr{\odot}_{\xi}^{G} \cap \mathfrak{k}^{\perp} \text { is a single } K \text {-orbit. }
$$

Although this conjecture seems rather bold, it does hold in many interesting cases and we have found no counterexamples to date. Moreover, we have recently proved a result that is only slightly weaker than the Orbit Conjecture.

Theorem 1. Let $K$ be a compact connected Lie group acting unitarily on $V$. Then $\left(K, H_{V}\right)$ is a Gelfand pair if and only if

(FOC) for every $\xi \in \mathfrak{k}^{\perp}, \mathscr{\odot}_{\xi}^{G} \cap \mathfrak{k}^{\perp}$ is a finite union of $K$-orbits.

A closely related result, due to V. Guillemin and S. Sternberg, can be found in [7]. They prove that if $G$ is compact, then $K \subset G$ is a Gelfand pair if and only if condition (FOC) holds generically. Theorem 1 does not, however, follow from the results in [7] since in our case, $G$ is not compact. We will outline our proof of this theorem below. Details of this work will appear elsewhere [1].

1.2. The moment map. One can write points $\xi \in \mathfrak{g}^{*}$ as $\xi=\left(\alpha, z_{\circ}, \lambda\right)$ where $\alpha \in \mathfrak{k}^{*}, z_{\circ} \in V, \lambda \in \mathbb{R}$, and $\xi(A, z, t)=\alpha(A)+\omega\left(z_{\circ}, z\right)+\lambda t$ for $A \in \mathfrak{k}$, $z \in V$, and $t \in \mathbb{R}$. One can verify that condition (OC) always holds for "nongeneric" orbits of the form $\mathscr{O}_{\left(\alpha, z_{\circ}, 0\right)}^{G}$. Moreover, one computes that for $\lambda \neq 0, \mathscr{O}_{(\alpha, 0, \lambda)}^{G} \cap \mathfrak{k}^{\perp}=\left\{(0, z, \lambda) \mid \tau(z) \in \mathscr{O}_{-2 \lambda \alpha}^{K}\right\}$ where $\tau: V \rightarrow \mathfrak{k}^{*}$ is the (unnormalized) moment map defined by

$$
\tau(z)(A):=\omega(z, A z)
$$

for $z \in V, A \in \mathfrak{k}$. Here $(A, z) \mapsto A z$ denotes the derived action of $\mathfrak{k}$ on $V$. A key property of $\tau$ is $K$-equivariance: $\tau(k z)=\operatorname{Ad}^{*}(k) \tau(z)$. In particular, $\tau$ maps $K$-orbits in $V$ to $\operatorname{Ad}^{*}(K)$-orbits in $k^{*}$. The above remarks lead to the following reformulations of conditions (OC) and (FOC).

- Condition (OC) holds if and only if $\tau$ is one-to-one on $K$-orbits.

- Condition (FOC) holds if and only if $\tau$ is finite-to-one on $K$-orbits. 
We can now remove the Heisenberg group from the picture, restating Theorem 1 as an equivalent result that provides a geometric criterion for multiplicity-free actions.

Theorem 2. The action of $K$ on $V$ is multiplicity-free if and only if the moment map $\tau: V \rightarrow \mathfrak{k}^{*}$ is finite-to-one on $K$-orbits.

The Orbit Conjecture is equivalent to the assertion that $K \times V \rightarrow V$ is a multiplicity-free action if and only if $\tau$ is one-to-one on $K$-orbits. In view of Theorem 2, this conjecture would be proved if one could show that $\tau$ is necessarily one-to-one on $K$-orbits whenever it is finite-to-one on $K$-orbits. Although we have been unable to show this, we have proved that if $\tau$ is finiteto-one on $K$-orbits, then $\tau$ is necessarily uniformly finite-to-one on $K$-orbits. This means that for some constant $M, \tau^{-1}\left(\mathscr{O}_{\alpha}^{K}\right)$ contains at most $M K$ orbits for all $\alpha \in \mathfrak{k}^{*}$. Thus, the finiteness result needed to complete the proof of the Orbit Conjecture would provide a geometric counterpart to the following lemma, which has a folklore status in the field.

Lemma 3. If the multiplicities of the representations of $K$ occurring in $\mathbb{C}[V]$ are finite and bounded, then $K \times V \rightarrow V$ is a multiplicity-free action.

1.3. Capelli actions. Let $I\left[\mathfrak{k}^{*}\right]$ denote the $\operatorname{Ad}^{*}(K)$-invariant polynomials on $\mathfrak{k}^{*}$ and $\mathbb{C}\left[V_{\mathbb{R}}\right]^{K}$ denote the $K$-invariant polynomials on the underlying real vector space $V_{\mathbb{R}}$ for $V$. Since $\tau$ is $K$-equivariant, we obtain an associated map $\tau^{*}: I\left[\mathfrak{k}^{*}\right] \rightarrow \mathbb{C}\left[V_{\mathbb{R}}\right]^{K}, \tau^{*}(p)(z):=p(\tau(z))$. Condition (OC) is equivalent to the assertion that $\tau^{*}\left(I\left[\mathfrak{k}^{*}\right]\right)$ separates $K$-orbits in $V$. Since $K$ is compact, one knows that the full algebra $\mathbb{C}\left[V_{\mathbb{R}}\right]^{K}$ of invariant polynomials always separates $K$-orbits. Thus we see that condition (OC) certainly holds whenever $\tau^{*}\left(I\left[\mathfrak{k}^{*}\right]\right)=$ $\mathbb{C}\left[V_{\mathbb{R}}\right]^{K}$. We say that $K \times V \rightarrow V$ is a Capelli action in this case. Theorem 2 shows that Capelli actions are always multiplicity free. Moreover, these provide an interesting class of Gelfand pairs that satisfy the Orbit Conjecture.

In [9] R. Howe and T. Umeda consider the "abstract Capelli problem" for actions of reductive complex algebraic groups. $K \times V \rightarrow V$ is a Capelli action if and only if the abstract Capelli problem for its complexification $K_{\mathrm{c}} \times V \rightarrow V$ has an affirmative answer. This means that the derived action of the universal enveloping algebra $\mathscr{U}\left(\mathfrak{k}_{c}\right)$ on $V$ yields a surjective map $d_{l}: \mathscr{Z} \mathscr{U}\left(\mathfrak{k}_{c}\right) \rightarrow$ $\mathscr{P} \mathscr{D}(V)^{K}$. Here $\mathscr{Z} \mathscr{U}\left(\mathfrak{k}_{c}\right)$ denotes the center of $\mathscr{U}\left(\mathfrak{k}_{c}\right)$, and $\mathscr{P} \mathscr{D}(V)^{K}$ is the algebra of $K$-invariant polynomial coefficient differential operators on $\mathbb{C}[V]$. This relation between Capelli actions and the abstract Capelli problem follows immediately from the commutative diagram (1) below.

The compact forms of many of Kac's irreducible multiplicity-free actions [10] are Capelli actions. From [9] one obtains a complete list of the irreducible Capelli actions (up to equivalence):

$\begin{array}{llll}U(n) & \text { on } \mathbb{C}^{n} & U(n) & \text { on } S^{2}\left(\mathbb{C}^{n}\right)(n \geq 2) \\ U(n) & \text { on } \Lambda^{2}\left(\mathbb{C}^{n}\right)(n \geq 2) & \mathbb{T} \times S O(n, \mathbb{R}) & \text { on } \mathbb{C}^{n}(n \geq 3) \\ U(n) \times U(m) & \text { on } \mathbb{C}^{n} \otimes \mathbb{C}^{m} & \mathbb{T} \times \operatorname{Sp}(n) & \text { on } \mathbb{C}^{2 n} \\ U(2) \times \operatorname{Sp}(n) & \text { on } \mathbb{C}^{2} \otimes \mathbb{C}^{2 n} & U(n) \times \operatorname{Sp}(4) & \text { on } \mathbb{C}^{n} \otimes \mathbb{C}^{8}(n \geq 4) \\ \mathbb{T} \times \operatorname{Spin}(7) & \text { on } \mathbb{C}^{8} & \mathbb{T} \times \operatorname{Spin}(10) & \text { on } \mathbb{C}^{16} \\ \mathbb{T} \times G_{2} & \text { on } \mathbb{C}^{7} & & \end{array}$


1.4. Spectrum of $\operatorname{Ind}_{K}^{G}\left(1_{K}\right)$. Suppose that $\left(K, H_{V}\right)$ is a Gelfand pair. The $K$-spherical representations of $G$ are precisely the spectrum of irreducible unitary representations weakly contained in the quasi-regular representation $\operatorname{Ind}_{K}^{G}\left(1_{K}\right)$ of $G$ on $L^{2}(G / K)$. The Orbit Method suggests that these representations should correspond to the integral coadjoint orbits in $\mathfrak{g}^{*}$ that meet $\mathfrak{k}^{\perp}$. Equivalently, one might expect that $\sigma \in \widehat{K}$ should occur in $\mathbb{C}[V]$ if and only if the corresponding coadjoint orbit in $\mathfrak{k}^{*}$ belongs to the image of the moment map $\tau: V \rightarrow \mathfrak{k}^{*}$. It is not hard to verify this for the standard action of the unitary group $U(V)$ (with respect to $\langle\cdot, \cdot\rangle$ ) on $V$. This fact together with functoriality of the moment map and a theorem of G. Heckman [8] concerning projections of coadjoint orbits establishes the following result.

Theorem 4. If $\sigma \in \widehat{K}$ occurs in $\mathbb{C}[V]$, then the corresponding coadjoint orbit in $\mathfrak{k}^{*}$ is contained in $\tau(V)$.

Although the converse does hold for some interesting examples, it is not true in general. $\tau(V)$ can contain integral coadjoint orbits that do not correspond to representations of $K$ occurring in $\mathbb{C}[V]$. This can happen even when $K \times V \rightarrow V$ is a Capelli action. The action of $U(n)$ on the symmetric 2-tensors $S^{2}\left(\mathbb{C}^{n}\right)$ provides an example of this phenomenon. It is known that the representations of $U(n)$ appearing in the (multiplicity-free) decomposition of $\mathbb{C}\left[S^{2}\left(\mathbb{C}^{n}\right)\right]$ are parameterized by Young's diagrams with all rows of even length. On the other hand, we have verified that the integral coadjoint orbits in the image of $\tau$ yield all representations parameterized by Young's diagrams of even size (total number of cells). Thus, the image of $\tau$ produces infinitely many representations of $U(n)$ that do not appear in $\mathbb{C}\left[S^{2}\left(\mathbb{C}^{n}\right)\right]$.

\section{OUTLINE OF PROOF OF THEOREM 2}

A key ingredient in our proof is the following commutative diagram relating $\tau^{*}$ to the action of the center of the complexified enveloping algebra of $k$ on $\mathbb{C}[V]$ by polynomial coefficient differential operators.

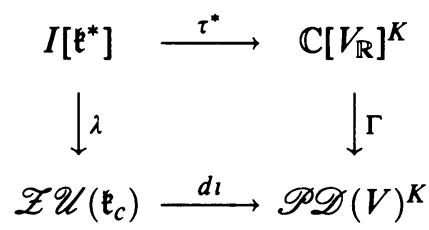

Here $\lambda$ is the symmetrization map (up to scalars), and $\Gamma$ takes elements of $V^{*}$ to multiplication operators and elements of $\bar{V}^{*}$ to differentiation operators. The horizontal maps are algebra maps, and the vertical maps are vector space isomorphisms.

Suppose that $K \times V \rightarrow V$ is a multiplicity-free action. It follows that $\mathscr{P} \mathscr{D}(V)^{K}$ is abelian [9] and hence, by a result of F. Knop [11], $\mathscr{P} \mathscr{D}(V)^{K}$ is finitely generated as a module over $d_{l}\left(\mathscr{Z} \mathscr{U}\left(\mathfrak{k}_{c}\right)\right)$. Although $\Gamma$ is not an algebra map, we do know that the terms of highest order in $\Gamma(p q)$ and $\Gamma(p) \Gamma(q)$ agree for any $p, q \in \mathbb{C}\left[V_{\mathbb{R}}\right]^{K}$. An induction argument on the total degree of operators in $\mathscr{P} \mathscr{D}(V)^{K}$ shows that $\mathbb{C}\left[V_{\mathbb{R}}\right]^{K}$ is a finitely generated $\tau^{*}\left(I\left[\mathfrak{k}^{*}\right]\right)$-module. 
Let $\gamma_{1}, \gamma_{2}, \ldots, \gamma_{\ell}$ be algebra generators for $\mathbb{C}\left[V_{\mathbb{R}}\right]^{K}$. Each $\gamma_{j}$ is algebraic over the fraction field of $\tau^{*}\left(I\left[\mathfrak{k}^{*}\right]\right)$. Suppose that $\gamma_{j}$ satisfies a polynomial equation of degree $d_{j}$ with coefficients $a_{i, j} \in \tau^{*}\left(I\left[\mathfrak{k}^{*}\right]\right)$. If $z_{\circ} \in V$ and $z \in$ $\tau^{-1}\left(\tau\left(K z_{\circ}\right)\right)$, then $\gamma_{j}(z)$ is a root of the degree $d_{j}$ polynomial with coefficients $a_{i, j}\left(z_{\circ}\right)$. Thus, $\gamma_{1} \times \gamma_{2} \times \cdots \times \gamma_{\ell}: V \rightarrow \mathbb{C}^{\ell}$ assumes at most $M:=d_{1} d_{2} \cdots d_{\ell}$ distinct values on $\tau^{-1}\left(\tau\left(K z_{\circ}\right)\right)$. As the level sets of $\gamma_{1}, \gamma_{2}, \ldots, \gamma_{\ell}$ are the $K$-orbits in $V$, this shows that $\tau$ is (uniformly) finite-to-one on $K$-orbits.

Next suppose that $\tau$ is finite-to-one on $K$-orbits and choose $\mathbb{R}$-valued generators $q_{1}, \ldots, q_{m}$ for $\tau^{*}\left(I\left[\mathfrak{k}^{*}\right]\right)$. For simplicity, we assume here that $q_{1}, \ldots$, $q_{m}$ are algebraically independent over $\mathbb{R}$. The level set of $q_{1} \times \cdots \times q_{m}$ through $z_{\circ} \in V$ is $\tau^{-1}\left(\tau\left(K z_{\circ}\right)\right)$, which is a finite union of $K$-orbits.

Let $z_{\circ}$ be a ("generic") point in $V$ where $D\left(q_{1} \times \cdots \times q_{m}\right)\left(z_{\circ}\right)$ has maximal rank. In fact, one can argue that this maximal rank is precisely $m$. Some open neighborhood of $z_{\circ}$ is foliated by $K$-orbits of codimension $m$. The orthogonal complement of the tangent space to $K z_{\circ}$ at $z_{\circ}$ is a dimension $m$ affine subspace $A$ of $V_{\mathbb{R}}$ that meets all $K$-orbits through points $z$ sufficiently close to $z_{\circ}$. Let $\tilde{q}_{j}:=\left.q_{j}\right|_{A} . \tilde{q}_{1}, \ldots, \tilde{q}_{m}$ are algebraically independent over $\mathbb{R}$ since a polynomial relation between the $\tilde{q}_{j}$ 's would imply (by $K$-invariance) a polynomial relation between the $q_{j}$ 's in a neighborhood of $z_{\circ}$. Now let $p$ be any $\mathbb{R}$-valued $K$-invariant polynomial on $V_{\mathbb{R}}$ and consider $\tilde{p}:=\left.p\right|_{A}$. By dimensional considerations, $\tilde{p}$ is algebraic over $\mathbb{R}\left(\tilde{q}_{1}, \ldots, \tilde{q}_{m}\right)$, and it follows by $K$-invariance that $p$ is algebraic over the fraction field of $\tau^{*}\left(I\left[\mathfrak{k}^{*}\right]\right)$. We conclude that $\mathbb{C}\left[V_{\mathbb{R}}\right]^{K}$ is a finitely generated $\tau^{*}\left(I\left[\mathfrak{k}^{*}\right]\right)$-module and hence that $\mathscr{P} \mathscr{D}(V)^{K}$ is finitely generated as a $d l\left(\mathscr{Z} \mathscr{U}\left(\mathfrak{k}_{c}\right)\right)$-module.

Let $\sigma \in \widehat{K}$ and $\mathscr{H}_{\sigma}$ be the space of highest weight vectors for $\sigma$ in $\mathbb{C}[V]$. The proof of Proposition 7.1 in [9] shows that $\mathscr{P} \mathscr{D}(V)^{K}$ must act irreducibly on $\mathscr{H}_{\sigma}$ when $\mathscr{P} \mathscr{D}(V)^{K}$ is a finitely generated $d l\left(\mathscr{Z} \mathscr{U}\left(\mathfrak{k}_{c}\right)\right)$-module. One argues that if $\left\{D_{1}, \ldots, D_{\ell}\right\}$ are generators for $\mathscr{P} \mathscr{D}(V)^{K}$ as a $d_{l}\left(\mathscr{Z} \mathscr{U}\left(\mathfrak{k}_{c}\right)\right)$-module and $p \in \mathscr{H}_{\sigma}$, then $\mathscr{H}_{\sigma} \subset \operatorname{Span}\left\{D_{1} p, \ldots, D_{\ell} p\right\}$. Thus the multiplicity of $\sigma$ in $\mathbb{C}[V]$ is at most $\ell$ for all $\sigma \in \widehat{K}$. Lemma 3 now implies that $K \times V \rightarrow V$ is a multiplicity-free action.

\section{ACKNOWLEDGMENT}

The authors thank Roger Howe for his many helpful comments concerning this work.

\section{REFERENCES}

1. C. Benson, J. Jenkins, R. Lipsman, and G. Ratcliff. A geometric criterion for Gelfand pairs associated with the Heisenberg group, preprint.

2. C. Benson, J. Jenkins, and G. Ratcliff, On Gelfand pairs associated with solvable Lie groups, Trans. Amer. Math. Soc. 321 (1990), 85-116.

3. G. Carcanno, A commutativity condition for algebras of invariant functions, Boll. Un. Mat. Ital. 7 (1987), 1091-1105.

4. L. Corwin and F. Greenleaf, Spectrum and multiplicities for restrictions of unitary representations in nilpotent Lie groups, Pacific J. Math. 135 (1988), 233-267.

5. I. M. Gelfand, Spherical functions on symmetric spaces, Dokl. Akad. Nauk USSR 70 (1950), 5-8; Amer. Math. Soc. Transl. Ser. 2, vol. 37, Amer. Math. Soc., Providence, RI, 1964, pp. 39-44. 
6. V. Guillemin and S. Sternberg, Geometric quantization and multiplicities of group representations, Invent. Math. 67 (1982), 515-538.

7. _ Multiplicity free spaces, J. Differential Geom. 19 (1984), 31-56.

8. G. J. Heckman, Projections of orbits and asymptotic behavior of multiplicities for compact connected Lie groups, Invent. Math. 67 (1982), 333-356.

9. R. Howe and T. Umeda, The Capelli identity, the double commutant theorem and multiplicity-free actions, Math. Ann. 290 (1991), 565-619.

10. V. Kac, Some remarks on nilpotent orbits, J. Algebra 64 (1980), 190-213.

11. F. Knop, A Harish-Chandra homomorphism for reductive group actions, preprint.

12. R. Lipsman, Orbit theory and harmonic analysis on Lie groups with co-compact nilradical, J. Math. Pure Appl. 59 (1980), 337-374.

13. Orbital parameters for induced and restricted representations, Trans. Amer. Math. Soc. 313 (1989), 433-473.

(C. Benson and G. Ratcliff) Department of Mathematics ANd Computer Science, UniverSity OF Missouri-ST. Louis, ST. Louis, MisSOURI 63121

$E$-mail address, C. Benson: bensonearch.umsl.edu

E-mail address, G. Ratcliff: ratcliffearch.umsl.edu

(J. Jenkins) Department of Mathematics and Statistics, State University Of New York at Albany, Albany, New York 12222

E-mail address: jwj710math.albany.edu

(R. Lipsman) Department of Mathematics, University of Maryland, College Park, MARYLAND 20742

E-mail address: rll@clio.umd.edu 